\title{
Enhancement of memory function in aged mice by a novel derivative of xanomeline
}

\author{
Yihui Cui ${ }^{1}$, Dong Wang ${ }^{1}$, Wen $\mathrm{Si}^{1}$, Wen $\mathrm{Lv}^{2}$, Yan $\mathrm{Niu}^{2}$, Xiaoping $\mathrm{Lei}^{2}$, Yinhe $\mathrm{Hu}^{1}$, Xiaohua Cao ${ }^{1}$ \\ 'Shanghai Institute of Brain Functional Genomics, The Key Laboratories of MOE and STCSM, East China Normal University, \\ 3663 Zhong Shan Road, Shanghai 200062, China; ${ }^{2}$ Peking University, School of Pharmaceutical Sciences, Beijing 100083, China \\ Cell Research (2008) 18:1151-1153. doi: 10.1038/cr.2008.292; published online 21 October 2008
}

\section{Dear Editor,}

In age-related cognitive deficits and Alzheimer's disease (AD), one of the most common pathological changes appears to be the loss of cholinergic neurons in the forebrain and the depletion of cortical cholinergic axons [1]. Accordingly, attempts have been made to generate therapies that aim to reverse the cognitive deficits associated with AD and aging by direct stimulation of mAChRs with specific agonists. Xanomeline is a well-known M1 agonist that has been shown to significantly improve cognitive function. However, side effects associated with hyper-activation of peripheral cholinergic systems have limited its clinical use [2]. Here, we describe our efforts to identify and characterize a novel derivative of xanomeline, 3-[3-(3-(3florophenyl)-2-propyn-1-ylthio)-1,2,5-thiadiazol-4-yl]1,2,5,6-tetrahydro-1-methylpyridine Oxalate (EUK1001).

EUK 1001, xanomeline, aceclidine and arecoline were synthesized in the laboratory of Dr Xiaoping Lei at the College of Medicine, Peking University. Xanomeline, aceclidine and arecoline were used as reference M1 agonists. The structures of EUK1001 and xanomeline are shown in Figure 1A.

We first evaluated the M1 agonist activities of compounds using a reporter gene assay. Xanomeline, aceclidine, arecoline and EUK1001 were found to show dose-dependent M1 agonist activity in reporter assays using HEK293/M1 cells, and no activity in negative control cell line, which was stably transfected only with the reporter gene construct. The EC50s were determined and are listed in Supplementary information, Table S1.

Correspondence: Xiaohua Cao

Fax: +86-21-62601953;

E-mail: xhcao@brain.ecnu.edu.cn
Among all four active compounds, EUK1001, whose EC50 value was the lowest (Supplementary information, Table S1), showed the best M1 agonist activity. Therefore, it was chosen for further characterization as a new lead compound for M1 agonists.

Xanomeline is known to impart significant deleterious side effects associated with peripheral cholinergic stimulation. To compare the side effect profile of EUK1001 with that of xanomeline, we injected aged mice intraperitoneally with $0.1,1.0$ or $10 \mathrm{mg} / \mathrm{kg}$ of the drug in saline solution. All treated mice were then observed and scored according to the presence of specific symptoms including salivation, lacrimation, tremors and diarrhea [3]. Scores of four animals were averaged in each dose group. At each tested dose, xanomeline produced more robust symptoms than EUK1001 during both the first $30 \mathrm{~min}$ (Figure 1B1) and the second $30 \mathrm{~min}$ (Figure 1B2). Based on these results, concentrations of 0.1 and $1.0 \mathrm{mg} / \mathrm{kg}$ EUK1001 were administered during the behavioral studies described below because of the low incidence of side effects.

To determine whether EUK1001 penetrates through the blood-brain barrier (BBB), the concentration of EUK1001 in plasma and brain samples was determined by a selective LC/MS/MS method.

Following i.p. administration to mice at the dose of $1 \mathrm{mg} / \mathrm{kg}$, the concentrations of EUK1001 in plasma and brain samples at three different time points were measured (Supplementary information, Table S2). The concentrations of EUK1001 in brain were higher than those in plasma at the same time point, indicating that EUK1001 can cross the BBB.

Based on the results of in vivo pharmacological screening and pharmacokinetics of EUK1001, aged C57 BL/6J mice were given daily i.p. injections with saline, $1.0 \mathrm{mg} / \mathrm{kg}$ xanomeline, $0.1 \mathrm{mg} / \mathrm{kg}$ EUK1001 
A

Xanomeline

EUK 1001<smiles>CCCCCCOc1nsnc1[C@H]1N=CCCN1C</smiles>

C (C1)

Training session

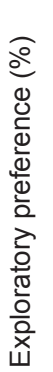

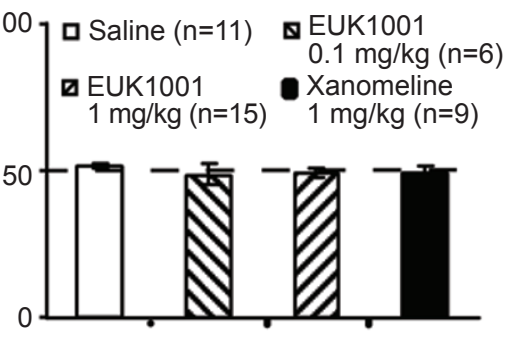

$(\mathrm{C} 2)$

Retention session

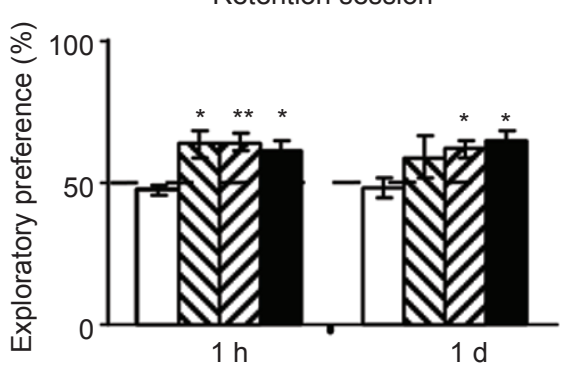

B (B1)
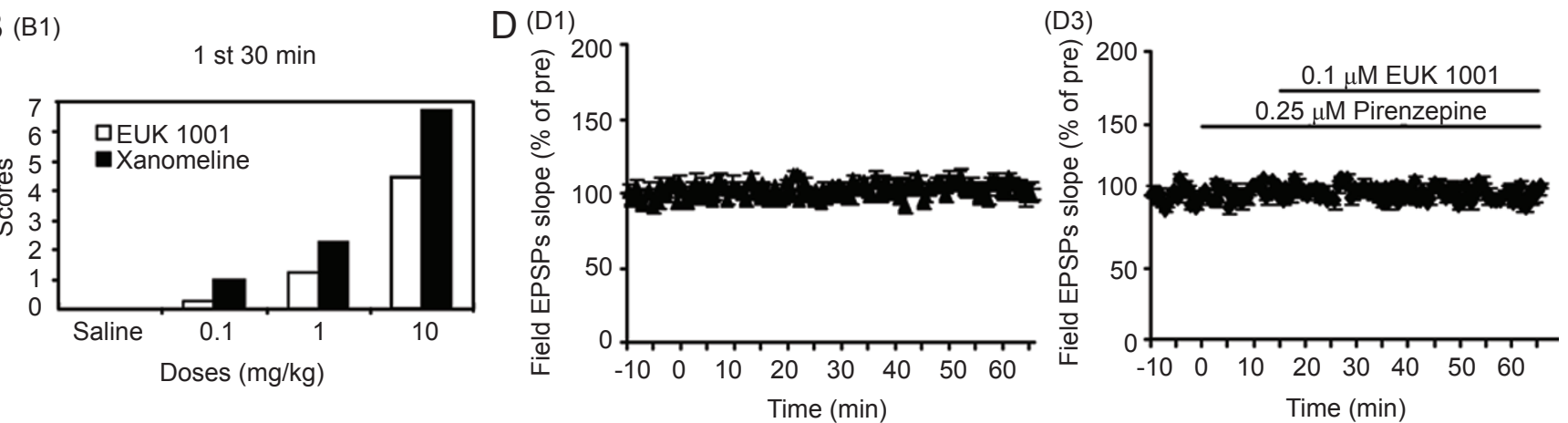

(B2)

(D2)

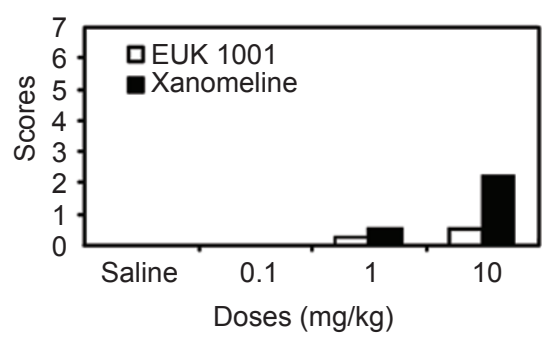

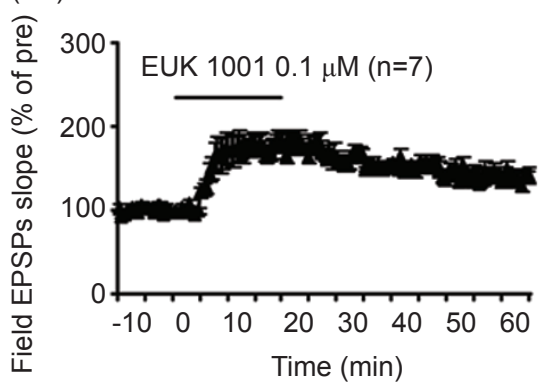

Figure 1 (A) Chemical structures of xanomeline and EUK1001. (B) Incidences of side effect during the first 30 min (B1) and the second $30 \mathrm{~min}$ (B2) following i.p. injection of xanomeline and EUK1001. (C) Effects of xanomeline and EUK1001 on cognition in aged mice. Xanomeline $(1 \mathrm{mg} / \mathrm{kg})$ and EUK1001 (0.1 or $1.0 \mathrm{mg} / \mathrm{kg})$ had no effects on basal exploratory behavior (C1), but improved the object recognition memory (C2). (D) Effects of EUK1001 on EPSPs of hippocampal CA3-CA1 in aged mice. (D1) EPSPs were evoked from CA3-CA1 of hippocampal slices under normal artificial cerebral spinal fluid (ACSF). $0.1 \mu \mathrm{M}$ EUK1001 induced the enhanced EPSP (D2), which can be completely blocked by $0.25 \mu \mathrm{M}$ pirenzepine (a selective M1 antagonist) (D3), but not by $0.25 \mu \mathrm{M}$ Methoctramine (a selective M2/M4 antagonist) (D4).

or $1.0 \mathrm{mg} / \mathrm{kg}$ EUK1001 for 15 days prior to behavioral testing. To first determine whether the drug treatments altered spontaneous activity of the mice, locomotor activities were assessed by measuring the distance traveled and the total time of movement during a 15min period. One-way ANOVA analysis revealed that neither xanomeline $(1.0 \mathrm{mg} / \mathrm{kg})$ nor EUK1001 ( 0.1 or 1.0 $\mathrm{mg} / \mathrm{kg}$ ) could significantly alter the time of movement (Supplementary information, Figure S1A, $F(3,37)=$ $1.63, P>0.05$ ) and the distance traveled (Supplementary information, Figure $\mathrm{S} 1 \mathrm{~B}, F(3,37)=1.51, P>0.05)$. This indicates that long-term treatment with the M1 receptor agonists elicits no obvious effect on locomotion or exploratory behavior of these mice.

To evaluate the effects of EUK 1001 on memory function in aged mice, we tested these mice in a novel object recognition task as described by Cao et al. [4]. As shown in Figure 1C1, treatment with xanomeline or EUK1001 did not significantly alter the time spent exploring objects during the training session compared to saline-injected controls $(F(3,37)=0.51, P>0.05)$. In contrast, post hoc analysis indicated that aged mice 
treated with EUK1001 (0.1 or $1.0 \mathrm{mg} / \mathrm{kg})$ or xanomeline $(1.0 \mathrm{mg} / \mathrm{kg})$ showed significantly enhanced exploratory preference for the novel object compared to saline control mice in the 1-h retention test (Figure 1C2, $F(3$, $37)=7.52, P<0.01)$. This enhanced memory persisted since treated mice also displayed elevated exploratory preference for the novel object in the 1-day retention test, although the difference was only significant for the higher dose groups $(1.0 \mathrm{mg} / \mathrm{kg}$ xanomeline and 1.0 $\mathrm{mg} / \mathrm{kg}$ EUK1001, Bonferroni test, $P<0.05$ ). These data demonstrate that both EUK1001 and xanomeline can significantly improve cognitive function in aged mice.

To understand the potential mechanism by which EUK1001 enhances memory, we studied its effects on plasticity of CA3-CA1 synapses of hippocampal slices from aged mice according to procedures described previously [4]. Superfusion with $0.1 \mu \mathrm{M}$ EUK1001 for $20 \mathrm{~min}$ induced a gradual, long-lasting increase in the slope of the field excitatory postsynaptic potential (fEPSP) evoked by stimulation of Schaffer collateral axons compared to the baseline slope $(139.7 \pm 1.12 \%, P<0.05$; Figure 1D2). This LTP-like response was blocked by 0.25 $\mu \mathrm{M}$ pirenzepine, a selective M1 antagonist, when applied 15 min prior to and during EUK1001 treatment (Figure 1D3). Notably, the robust potentiation of the EPSP slope induced by $0.1 \mu \mathrm{M}$ EUK1001 was not affected by 0.25 $\mu \mathrm{M}$ Methoctramine, a selective antagonist of M2/M4 receptors $(142.3 \pm 3.75 \%, \mathrm{n}=5, P<0.05$ vs baseline, $P=0.82$ vs LTP induced by $0.1 \mu \mathrm{M}$ EUK1001; Figure 1D4). Together, these results suggest that EUK1001 can enhance LTP in an M1 receptor-dependent fashion.

EUK1001 was selected as a leading candidate because it was found to elicit potent M1 receptor agonist activity. Importantly, we found that, at all doses tested, the aged mice treated with EUK1001 were significantly less prone to overt episodes of side effects than those mice treated with equivalent doses of xanomeline.

Compared to saline- injected controls, aged mice injected i.p. with either 0.1 or $1.0 \mathrm{mg} / \mathrm{kg}$ EUK1001 for 15 days prior to the task showed significantly enhanced preference for the novel object in the 1-h retention test. Similar results were found when $1.0 \mathrm{mg} / \mathrm{kg}$ xanomeline was administered for 15 days. Furthermore, mice injected with $1.0 \mathrm{mg} / \mathrm{kg}$ of either xanomeline or EUK 1001 exhibited enhanced preference for the novel object in the 1-day retention test.

The modulatory effects of acetylcholine on hippocampal synaptic plasticity have been documented previously [5, 6]. In order to understand the mechanism of EUK1001induced enhancement of memory function in aged mice, we investigated its effects on hippocampal synaptic plasticity. Bath application of EUK1001 to hippocampal slice preparations induced an LTP-like response, which was blocked by pirenzepine, a selective M1 antagonist. This chemically induced LTP has been postulated to involve the enhancement of synaptic transmission [7]. Thus, our experiments provide a physiological correlation between enhancement of memory function and synaptic plasticity.

In summary, our results suggest that EUK1001 exhibits higher affinity for the human M1 receptor than xanomeline and produces significantly less deleterious side effects. In addition, EUK1001 is capable of increasing recognition memory in aged mice and inducing LTP at CA1-CA3 synapses in hippocampal slices. Thus, EUK1001 may represent a promising therapeutic agent for the treatment of AD and age-related memory disorders.

\section{Acknowledgments}

This research was supported by the National Basic Research Program of China (973 Program) (NO2003CB716605) from the Ministry of Science and Technology, National Natural Science Foundation of China (NO30670682, NO30640068), and grants from Shanghai Science and Technology Commission (05DJ14007, 06DZ19003).

\section{References}

1 Schliebs R, Arendt T. The significance of the cholinergic system in the brain during aging and in Alzheimer's disease. $J$ Neural Transm 2006; 113:1625-1644.

2 Bodlick NC, Offen WW, Levey AI, et al. Effects of xanomeline, a selective muscarinic receptor agonist, on cognitive function and behavioral symptoms in Alzheimer's disease. Arch Neurol 1997; 54:465-473.

3 William SM, Kenneth AB, Colleen D, et al. Development of CDD-0102 as a selective M1 agonist for the treatment of Alzheimer's disease. Drug Dev Res 2002; 57:200-213.

4 Cao XH, Cui Z, Feng R, et al. Maintenance of superior learning and memory function in NR2B transgenic mice during ageing. Eur J Neurosci 2007; 25:1815-1822.

5 Sokolov MV, Kleschevnikov AM. Atropine suppresses associative potentiation in the hippocampus. Brain Res 1995; 672:281-284.

6 Boddeke EW, Enz A, Shapiro G. SDZ ENS 163, a selective muscarinic M1 receptor agonist, facilitates the induction of long-term potentiation in rat hippocampal slices. Eur $J$ Pharmacol 1992; 222:21-25.

7 Guo X, Lester RA. $\mathrm{Ca}^{2+}$ flux and signaling implications by nicotinic acetylcholine receptors in rat medial habenula. $J$ Neurophysiol 2007; 97:83-92.

(Supplementary information is linked to the online version of the paper on the Cell Research website.) 